\title{
Square Root Raised Cosine Fractionally Delaying Nyquist Filter - Design and Performance Evaluation
}

\author{
Ewa Hermanowicz and Mirosław Rojewski
}

\begin{abstract}
In this paper we propose a discrete-time FIR (Finite Impulse Response) filter which is meant to be applied as a square root Nyquist filter and fractional delay filter simultaneously. The filter enables to substitute for a cascade of square root raised cosine (SRRC) Nyquist filter and fractional delay filter in one device/algorithm. The aim is to compensate for transmission delay in digital communication system. Performance of the filter in the role of a matched filter is evaluated using a newly defined energetic ISI (Intersymbol Interference) measure and ability of the filter to completely eliminate the ISI involved by fractional delay of symbol shaping filter in transmitter or by channel delay. Considerations and results of the contribution are documented by suitable eye-diagrams and the SRRC filter responses.
\end{abstract}

Keywords - SRRC (Square Root Raised Cosine) Nyquist filter, special FIR (Finite Impulse Response) filter design, ISI (Intersymbol Interference), fractional delay filter

\section{INTRODUCTION}

$\mathbf{N}$ YQUIST filters [1], [2], [3], [4], [5], [6], [7], [8], [9], [10], [11], [12], [13], [14] are very important modules inherent in up-to-date data transmission systems through a band limited channel. The most often met of this kind of filter is SRRC -Square Root Raised Cosine filter. It is normally applied in transmitter side as a symbol signal shaping filter and in receiver side as a filter matched to elementary signal conveying a symbol of digital data. However, in this paper the SRRC in transmitter side is not only a model of a symbol signal shaping filter but also it models the fractional subsample group delay $d, d \in[-1 / 2,1 / 2]$, introduced by the transmission channel, measured in samples (Sa). Consequently, in the receiver, the matched filter should have the fractional sample delay of the opposite sign relative to the transmitter delay, imposed by the system of symbol synchronization, in order to compensate for the delay $d$.

The scope of the paper is as follows. An original (with the best authors' knowledge) way of designing the SRRC FIR (Finite Impulse Response) filter is proposed in Sect. II. It is a filter aggregating two features: ISI (Intersymbol Interference) free condition [1], [2], [3], [4], [5] and fractional time delaying [6], [7], [8], [9], [12] the signal under processing. In Sect. III we give an infographic documentation typical of this kind of filters performance with coefficients normalized to unit energy. Section IV is devoted to the eye-diagram representation. In order to evaluate the performance of the SRRC filter in the role of a matched filter in Section $\mathrm{V}$ we

The authors are with Faculty of Electronics, Telecommunication and Informatics, Technical University of Gdansk, Poland (e-mails: hewa@eti.pg.gda.pl, ro@sound.eti.pg.gda.pl). define an energetic ISI measure characterizing the filter. In Section VI we summarize and conclude the contribution.

\section{BLOCK-SCHEME OF PROCESSING}

Figure 1 shows the block scheme illustrating the processing used in our experiments. Here instead of using a single raised cosine (RC) Nyquist filter of fractional sample group delay modeling overall signaling channel as it was in [12], a SRRC filter of fractional sample (or subsample) delay [6] is used at both transmitter and the receiver.

It is assumed that the system input is a synthetic irredundant quadrature phase shift (QPSK) symbol train

$$
\left.c[m]=(1+j) \exp \left(j \frac{\pi}{2} i[m]\right)=\sqrt{2} \exp \left(j\left(\frac{\pi}{4}+\frac{\pi}{2} i[m]\right)\right)\right)
$$

where $m=\cdots, 0,1,2, \cdots$, expressed by one sample (Sa) per symbol (1 Sa/symbol) and $i[m] \in\{0,1,2,3\} \forall m$ are random independent numbers. The symbol sequence $c[m]$ is interpolated by a factor of $L$ using an adequate SRRC FIR filter denoted $G_{d}$. As a result this gives a signal (sample train)

$$
\left\{v_{d}[n]\right\}=G_{d}\left\{c_{L}[n]\right\}=G_{d} L \uparrow\{c[m]\} ; \quad n=\cdots, 0,1,2, \cdots
$$

Here symbol $L \uparrow$ stands for $L$-fold sample rate expander (zero inserter) followed by interpolating filter $G_{d}$. The linear operator $G_{d}$ is meant to describe the frequency response $G_{d}\left(e^{j \omega}\right)$ of the transmitter shaping SRRC filter in transmitter side of communication path [7] and $g_{d}[n]$ is the impulse response of this filter having fractional group delay $d$ Sa introduced by transmission channel. Here $\omega \in[-\pi, \pi)$ in $\mathrm{rad} / \mathrm{Sa}$ is angular (radian) frequency. Further on we shall use also normalized frequency $f=\omega /(2 \pi) \in[-1 / 2,1 / 2)$ which is understood as a part of sampling rate. Hence $G_{-d}\left(e^{j \omega}\right)$ and $g_{-d}[n]$ stand for the frequency response and impulse response of the matched filter, respectively, that maximizes signal to noise ratio (SNR), operating in receiver side and having fractional delay $-d$ thus compensating the channel delay. The signal $v_{d}[n]$ is received from transmission or propagation channel (here noiseless and of flat group delay) thus shaped by the SRRC and delayed by $d$. Here 
$\left\{w_{d d}[n]\right\}=G_{-d}\left\{v_{d}[n]\right\}=G_{-d} G_{d}\left\{c_{L}[n]\right\}=H_{d d}\left\{c_{L}[n]\right\} ;$

$n=\cdots, 0,1,2, \cdots$

is the received complex envelope in which the fractional delay $d$ is compensated and whose instantaneous phase is denoted by $\varphi_{d d}[n]$.

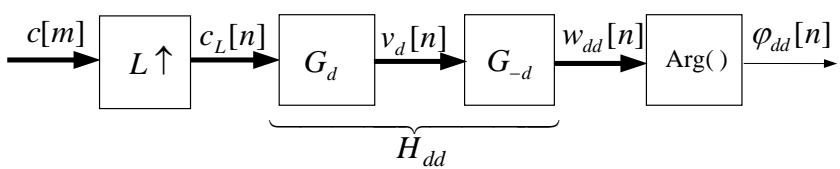

Fig. 1. Block scheme of processing used in experiments; thin arrow stands for real-valued signal and thick arrow indicates complex-valued signal; operator $H_{d d}$ marks an equivalent for the cascade of $G_{d}$ and $G_{-d}$ filters.

The ideal prototype of the SRRC filter here is a classical analog SRRC Nyquist filter with the roll-off factor $\alpha \in[0,1]$ for the spectrum meaning an intentional excess bandwidth, whose impulse response (both sides infinite) in the continuous time domain is expressed as [5], [7]

$$
g(t)=\frac{\sin \left[\pi \frac{t}{T}(1-\alpha)\right]+4 \alpha \frac{t}{T} \cos \left[\pi \frac{t}{T}(1+\alpha)\right]}{\pi \frac{t}{T}\left[1-\left(4 \alpha \frac{t}{T}\right)^{2}\right]}
$$

for $t \in(-\infty, \infty)$ with exceptions that

$$
g(t)=\left\{\begin{array}{c}
(1-\alpha)+\frac{4 \alpha}{\pi} ; t=0 \\
\frac{\alpha}{\sqrt{2}}\left[\left(1+\frac{2}{\pi}\right) \sin \left(\frac{\pi}{4 \alpha}\right)+\left(1-\frac{2}{\pi}\right) \cos \left(\frac{\pi}{4 \alpha}\right)\right] ; \frac{t}{T}= \pm \frac{1}{4 \alpha}
\end{array}\right.
$$

due to the consequence of del'Hospital rule.

A discrete-time version of the above formula one obtains by substituting

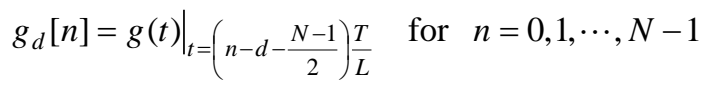

to achieve the impulse response of a novel SRRC FIR filter involving fractional group delay $d$ and transport (bulk) time delay $D=\frac{N-1}{2}$ (both denominated in Sa)

$$
\begin{aligned}
& g_{d}[n]= \frac{\sin \left[\pi \frac{n-d-D}{L}(1-\alpha)\right]}{\pi \frac{n-d-D}{L}\left[1-\left(4 \alpha \frac{n-d-D}{L}\right)^{2}\right]} \\
&+\frac{4 \alpha \frac{n-d-D}{L} \cos \left[\pi \frac{n-d-D}{L}(1+\alpha)\right]}{\pi \frac{n-d-D}{L}\left[1-\left(4 \alpha \frac{n-d-D}{L}\right)^{2}\right]}
\end{aligned}
$$

Such a problem has not been posed and developed in the literature yet. Further on the properties of the $G_{d}$ filter for $d \in[-0.5,0.5]$ is documented and illustrated.

\section{NOVEL FILTERS CHARACTERISTICS}

Below we have gathered some examples of the novel SRRC filter $G_{d}$ of fractional delay $d$ value from the previous Section.

a)

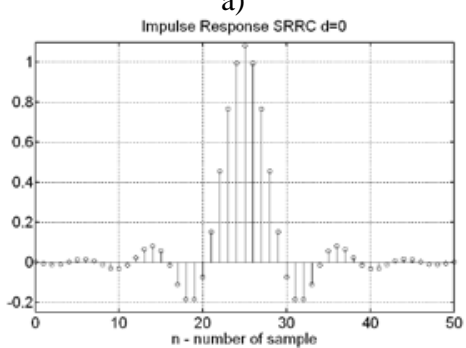

b)

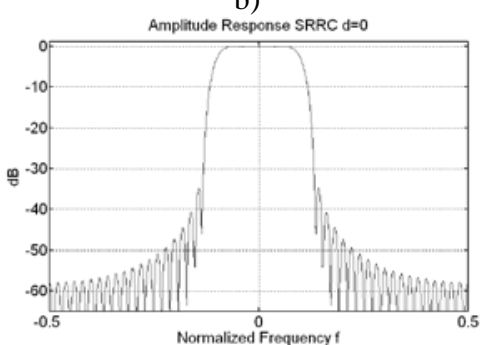

c)
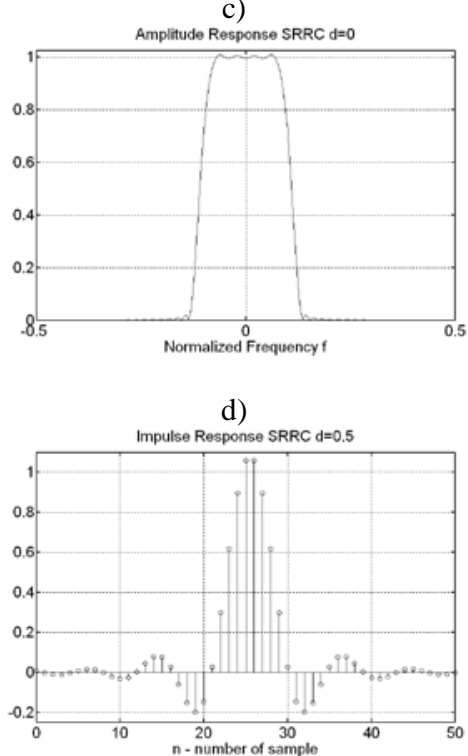

e)

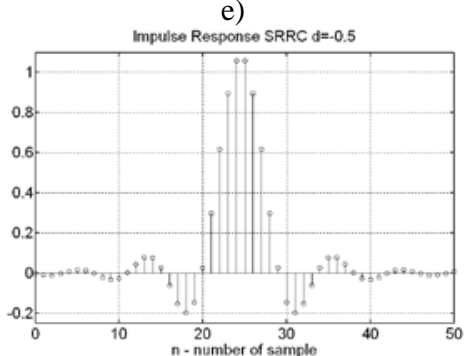

Fig. 2. Impulse response (a) of the SRRC Nyquist filter $G_{d}$ of length $N=51$ and fractional delay $d=0$, (b) the filter amplitude response for $d=0$ in $\mathrm{dB}$ and (c) in linear scale as well as impulse responses for $d= \pm 0.5$ (d, e) where they are sampled at the midpoints of sample interval as in (a) for $d=0$ (cf. (2)). 
The symbol interval in our examples equals $5 \mathrm{Sa}$, hence the interpolation factor in the scheme in Fig. 1 is $L=5$ and typical roll-off factor $\alpha=0.3$. Length of this filter is generally set to be odd [5], [6], [7], [8] as given by formula $N=2 \lambda L+1$ where $\lambda$ stands for the number of zero-crossings in the impulse response (5) tails before an after the maximal magnitude coefficient appearing for $n=(N-1) / 2=D$. In our experiments here we use $\lambda=5$, thus $N=51$ and $D=25$.

a)

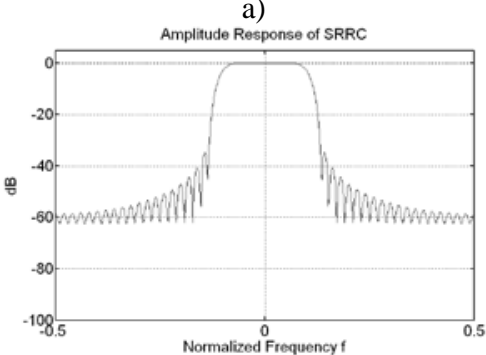

b)
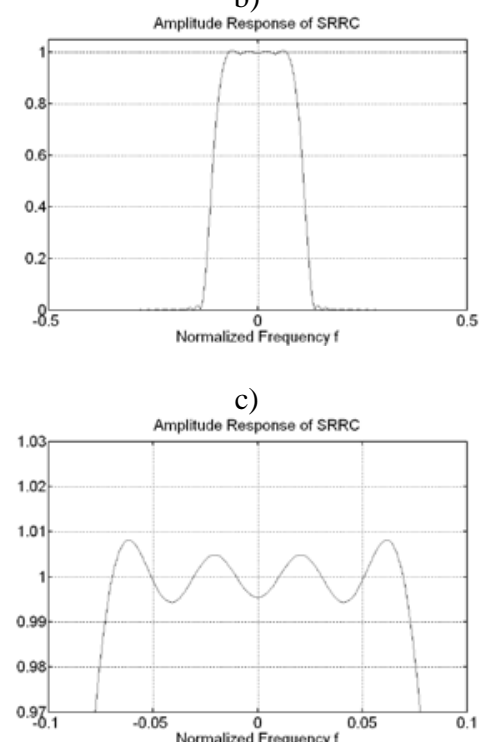

d)

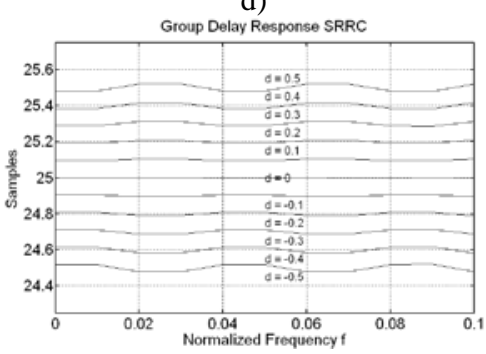

Fig. 3. Amplitude response of the SRRC filter of $d= \pm 0.5$ in $\mathrm{dB}$ (a) and in linear scale (b), and in the linear scale zoomed (c), and group delay responses in passband (d) between $d=0$ and $d=+0.5$ as well as between $d=0$ and $d=-0.5$.

The impulse responses of the SRRC filter for $d=0$ and for $d= \pm 0.5$, and amplitude response $\left|G_{d}\left(e^{j \omega}\right)\right|_{\omega=2 \pi f}$ for $d=0$ are depicted in Fig. 2.
In Fig. 3a, b, c we have presented the amplitude response of the SRRC filter with $d= \pm 0.5$. This is very similar to that in Fig. 2 for $d=0$.

a)

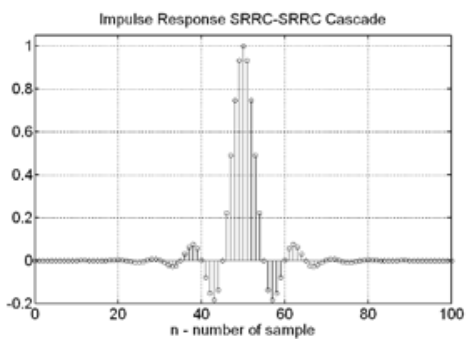

b)

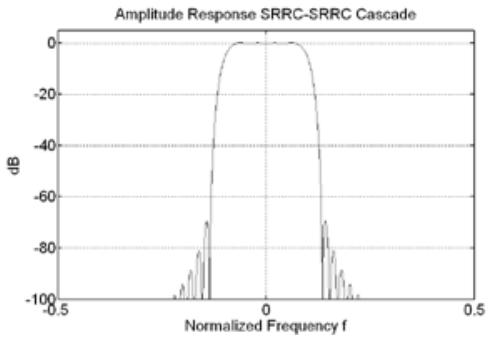

c)

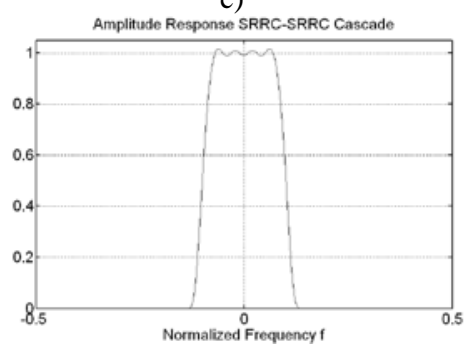

d)

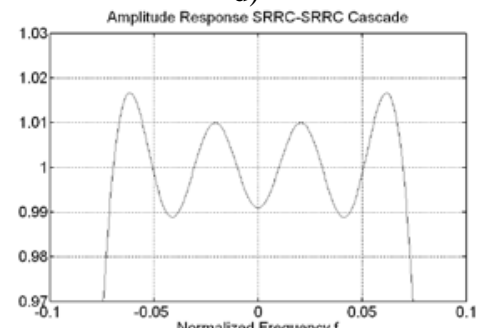

Fig. 4. Impulse response and amplitude response of the filter $H_{d d}$ (see Fig. 1) for the shaping SRRC filter of $d= \pm 0.5$ in transmitter cascaded with the matched SRRC filter in the receiver of $d=\mp 0.5$ named SRRC-SRRC Cascade; the $H_{d d}$ filter length is $2 N-1=101$ and its group delay response is flat and equal $N-1=50$.

The term "fractional delay" mentioned in the paper title denotes the fractional part of group delay response $\tau_{d}(\omega)$ of the filter $G_{d}$ defined as

$$
\tau_{d}(\omega)=-\frac{\mathrm{d}\left[\arg G_{d}\left(e^{j \omega}\right)\right]}{\mathrm{d} \omega}=-\frac{\mathrm{d} G_{d}\left(e^{j \omega}\right) / \mathrm{d} \omega}{G_{d}\left(e^{j \omega}\right)}
$$


and measured in Sa. This way defined group delay responses of SRRC filters discussed here are collected in Fig. 3d. The "arg” in (7) stands for the unwrapped argument of a complex function.

From the group delay responses in Fig. 3d we can see that they are slightly rippled and symmetric (here shown in pass band) around bulk delay $D=25$ where $d=0$.

Now, let us focus on $H_{d d}$ filter replacing in Fig. 1 the cascade of two (shaping and matched) SRRC filters of the same magnitude but with opposite value of parameter $d$; formally

$$
H_{d d}=G_{-d} G_{d}=G_{d} G_{-d}
$$

Hence amplitude response of the cascade equivalent filter is

$$
\left|H_{d d}\left(e^{j \omega}\right)\right|=\left|G_{d}\left(e^{j \omega}\right)\right|^{2}=\left|G_{-d}\left(e^{j \omega}\right)\right|^{2}
$$

For exemplary $N=51$ one obtains the $H_{d d}$ symmetric FIR filter of length $2 N-1=101$ (see Fig. 4a) whose group delay response is flat and equal exactly $2 D=N-1=50$. It means that the fractional delay $d$ was compensated by the cascade of filters with an opposite sign of $d$. The amplitude response of the $H_{d d}$ filter is presented in Fig. 4b, c and d, in logaritmic (dB) scale and in linear scale. The amplitude response of this filter is similar to that of respective SRRC amplitude response from Fig. 3. Comparing the figures we can see in Fig. 4 two times greater ripples in passband and two times smaller ripples in stopband than in Fig. 3. This observation could be read out from the relation (9). But such visual inspection of the above characteristics does not allow to evaluate how much the residual ISI of the $G_{-d} G_{d}$ cascade is left in received complex envelope $w_{d d}[n]$.

\section{EYE DIAGRAMS}

An estimation at the first glance of the residual ISI can be obtained from classical eye diagrams of the complex envelope $w_{d d}[n]$ Cartesian components.

In Fig. 5 we have shown the eye diagrams [5] of in-phase component (real part) of received complex envelope $w_{d d}[n]$ for the scenario depicted in Fig. 1 - i.e. obtained as a result of filtering described by (1c) whose equivalent amplitude response is shown in Fig. 4. We can see in Fig. 5 that in the zoomed residual ISI eye-diagram amplitude jitter [5] is approximately $5 \%$ of its true value in maximal vertical eye opening instant. (a)

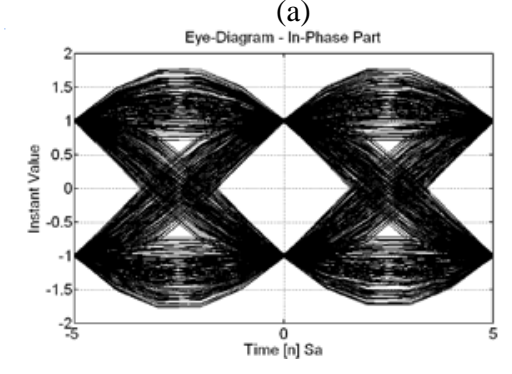

(b)

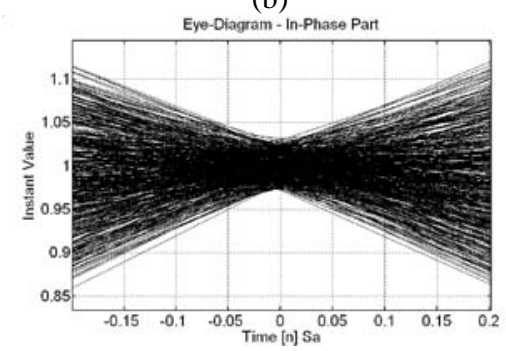

(c)

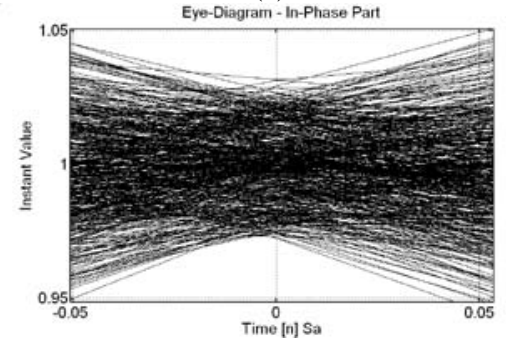

Fig. 5. Eye-diagram (a) of complex envelope $w_{d d}[n]$ as a response of cascaded transmitter SRRC filter of $d= \pm 0.5$ and matched SRRC fiter $d=\mp 0.5$ in the receiver; excited by symbol train (1a); both SRRC filters are of length $N=51$; below is maximal vertical opening instant (b) and (c) zoomed from the part (a) of the same figure.

For comparison the eye diagrams at the output of the shaping SRRC filter (denoted by $G_{d}$ in Fig. 1) excited by (1) are presented in Fig. 6 for $d=0$ (a), $d=0.5$ (b) and $d=-0.5$ (c).

(a)

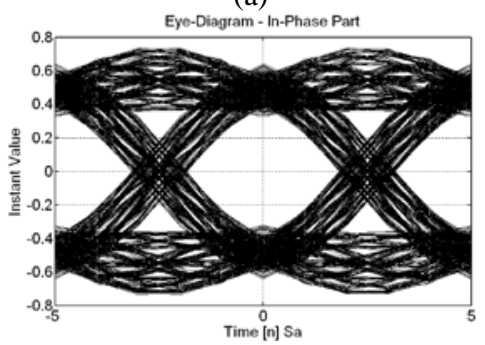

(b)

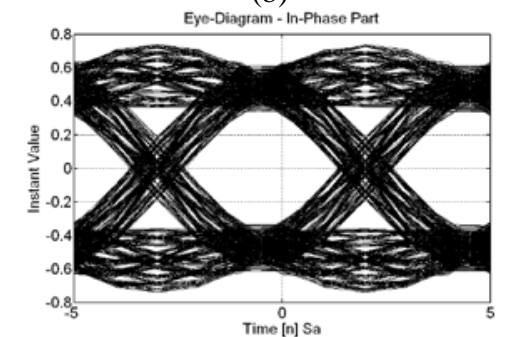

(c)

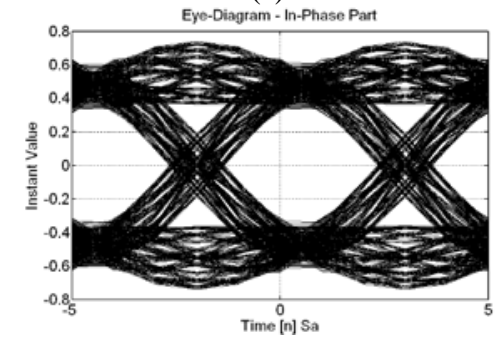

Fig. 6. Eye diagrams at the output of the shaping SRRC (denoted by $G_{d}$ in Fig. 1) of length $N=51$ excited by (1a); (a) $d=0$, (b) $d=0.5$ and (c) $d=-0.5$. 
From the above three eye-diagrams shown in Fig. 6 the fractional delaying effect of $G_{d}$ filter is evident and also in Fig. 6 the eye-diagram blurring in maximum opening instant can be estimated as many times greater than after matched filter $G_{-d}$ in Fig. 5a.

\section{ISI EVALUATION MEASURE}

The quantitative ISI measure involved by 1/L-band filter, approaching the Nyquist RC filter, can be derived estimating how much (in what degree) the impulse response $h[n]$ of this filter does not fulfill the Nyquist condition [13], [14]. For such a FIR filter of length $N$ odd and nearly symmetric (i.e. of the coefficient having maximal magnitude for $n=(N-1) / 2$ and of the same number $\lambda$ of zero-crossings in each of its tails) this Nyquist condition can be written as follows

$$
h[n]=\left\{\begin{array}{c}
1, \quad n=(N-1) / 2 \\
0, \quad n=(N-1) / 2 \pm k L, \quad k=1,2, \cdots, \lambda \\
\text { arbitrary for } n \neq(N-1) / 2 \pm k L
\end{array}\right.
$$

Hence, in this Section we give a new quantitative insight into ISI evaluation measure. More generally let $h[n]$, $n=0,1, \cdots N-1$ be the impulse response of FIR interpolation filter (or decimation filter, Nyquist RC filter, SRRC filter, a cascade of two SRRC filters, ...) of $1 / L$ bandwidth and of odd length $N$ (6) with $L=2,3,4, \cdots$ and $\lambda=2,3,4, \cdots$. The measure of ISI relative quantity introduced by the filter we define by the formula

$$
I=\sum_{k=1}^{\lambda} \frac{\left\{h\left[\frac{N-1}{2}+k L\right]+h\left[\frac{N-1}{2}-k L\right]\right\}}{h\left[\frac{N-1}{2}\right]}
$$

This measure is valid for a particular input having the form of unipolar equivalued symbol train. The value $I$ in (11) is a small number, whose modulus can be expressed in percent $|I|$ $\%$. However, it is more adequate to use root mean square measure of ISI quantity

$$
I_{\text {rms }}=\frac{\left(\sum_{k=1}^{\lambda}\left\{\left|h\left[\frac{N-1}{2}+k L\right]\right|^{2}+\left\lfloor\left. h\left[\frac{N-1}{2}-k L\right]\right|^{2}\right\}\right)^{\frac{1}{2}}\right.}{\left|h\left[\frac{N-1}{2}\right\rfloor\right|}
$$

The $I_{\text {rms }}$ is a measure which is consistent with eye-diagram blurring discussed in previous Section. In Table I we can see about 10 (and more) times compression of $I_{\text {rms }}$ for the cascade SRRC in $H_{d d}$ ( $G_{d}$ and $G_{-d}$ in Fig. 1) relative to $G_{d}$ or $G_{-d}$ inspected solely. This compression ratio remains in agreement with how much the eye-diagram in Fig. 5 is concentrated by the matched filter.

TABLE I

Table I. ROOT MEAN SQUARE (RMS) ISI MEASURES FOR SRRC AND FOR THE Cascade of Transmission $G_{d}$ (Shaped) ANd Receiver $G_{-d}$ (MATCHED) SRRC FILTERS OF FRACTIONAL DELAY D

\begin{tabular}{|c|l|l|}
\hline$d$ & $\begin{array}{l}I_{\text {rms }} \\
\text { for SRRC in \% }\end{array}$ & $\begin{array}{l}I_{\text {rms }} \\
\text { for SRRC-SRRC Cascade in \% }\end{array}$ \\
\hline 0 & 13.12 & 1.36 \\
\hline \pm 0.1 & 13.25 & 1.36 \\
\hline \pm 0.2 & 13.66 & 1.36 \\
\hline \pm 0.3 & 14.33 & 1.35 \\
\hline \pm 0.4 & 15.26 & 1.33 \\
\hline \pm 0.5 & 16.43 & 1.32 \\
\hline
\end{tabular}

Another expression for ISI measure, this time an energetic measure $I_{e}$, is given by

$$
I_{e}=\frac{\sum_{k=1}^{\lambda}\left\{\left|h\left[\frac{N-1}{2}+k L\right]\right|^{2}+\left|h\left[\frac{N-1}{2}-k L\right]\right|^{2}\right\}}{\left|h\left[\frac{N-1}{2}\right]\right|^{2}}=I_{\mathrm{rms}}^{2}
$$

Both this measures (12) and (13) can be expressed in percent or on logarithmic scale. Moreover, reciprocal of these measures have to be treated as SNR i.e. ratio of the symbol energy to energy of noise "produced" by intentional or residual ISI.

TABLE I

ENERGETIC ISI MEASURES FOR SRRC AND FOR THE CASCADE OF TRANSMission $G_{d}$ (SHAPED) AND RECEIVER $G_{-d}$ (MATCHED) SRRC FILTERS OF FRACTIONAL DELAY D

\begin{tabular}{|c|l|l|}
\hline$d$ & $\begin{array}{c}I_{e} \\
\text { for SRRC in } \%\end{array}$ & $\begin{array}{l}I_{e} \\
\text { for SRRC -SRRC Cascade in \% }\end{array}$ \\
\hline 0 & 1.72 & $1.86 \cdot 10^{-2}$ \\
\hline \pm 0.1 & 1.76 & $1.855 \cdot 10^{-2}$ \\
\hline \pm 0.2 & 1.87 & $1.839 \cdot 10^{-2}$ \\
\hline \pm 0.3 & 2.05 & $1.814 \cdot 10^{-2}$ \\
\hline \pm 0.4 & 2.33 & $1.779 \cdot 10^{-2}$ \\
\hline \pm 0.5 & 2.7 & $1.7354 \cdot 10^{-2}$ \\
\hline
\end{tabular}

In Table II we can see about 100 (and more) times compression of $I_{e}$ for the cascade SRRC in $H_{d d}\left(G_{d}\right.$ and $G_{-d}$ in Fig. 1) relative to $G_{d}$ or $G_{-d}$ inspected solely. This number represents processing gain of receiver due to an application of the matched filter $G_{-d}$ used in Fig. 1. This justifies the sense and usefulness of ISI measures proposed in this contribution.

\section{CONCLUSIONS}

The subject of investigations described in this paper was introduced in [12], [8] as a special (here Nyquist) discretetime filter of fractional group delay [6]. The concept of enrichment the Nyquist RC filter, formerly explained in [12], 
by this extra (and important in applications) feature, without making complicated its structure and design, was transferred here from RC to SRRC filters. On the example of typical isochronous system of data transmission applying QPSK signals, the practical usefulness of the novel kind of the proposed non-recursive filter as a time-synchronized matched filter enabling the receiver optimal ISI elimination was experimentally proven. Operation of the new filter, both in roles of the shaping filter as well as the matched filter, was illustrated using suitable eye-diagrams. A new ISI (rms and energetic) measures involved by $1 / L$-band filter were defined and applied to performance evaluation of the shaping filter and the cascade of the shaping filter and the matched filter. Our experiments revealed that the ISI involved by the SRRC shaping filter due to its fractional delay was almost completely eliminated by the SRRC matched filter of fractional delay but with opposite sign relative to the shaping filter.

\section{REFERENCES}

[1] D. W. Tufts, „Nyquist's problem - The joint optimization of transmitter and receiver in pulse amplitude modulation”, Proc. IEEE, vol. 53, pp. 248-259, Mar. 1965.

[2] L. E. Franks, „Futher results on Nyquist's problem pulse transmission”, IEEE Trans. Commun. Technology, vol. COM16, pp. 337-340, Apr. 1966.

[3] R. W. Lucky, J. Salz, and E. S. Weldon, Jr., Principles of Data Communication, McGraw-Hill, New York, 1968.
[4] L. E. Franks, Signal Theory, Prentice Hall, Englewood Cliffs, New Jork, 1969.

[5] J. D. Gibson (Ed), The Communications Handbook, CRC Press, 2002, 2nd Ed., Chapter 23.7, p. 23-5.

[6] E. Hermanowicz, Special Discrete-Time Filters and Applications, EXIT, Warsaw, 2005.

[7] F. J. Harris, Multirate Signal Processing for Communication Systems, Prentice Hall PTR, 2004.

[8] C-C. Tseng, „Designs of fractional delay filter, Nyquist filter, lowpass filter and diamond-shaped filter”, Signal Processing, vol. 87, pp. 584-601, 2007.

[9] F. Mintzer, „On half-band, third-band and Nth band FIR filters and their design”. IEEE Trans. on Signal Processing, vol. ASSP-30, No 5, pp. 734-738, 1982.

[10] P.P. Vaidyanathan, Multirate Systems and Filter Banks, Prentice Hall, 1993.

[11] D.R. Smith, Digital Transmission Systems, Kluwer Academic Publishers, 3rd Ed., Chaper 5, p. 5.6, pp. 285-288, 2003.

[12] E. Hermanowicz and M. Rojewski, „On designing a Nyquist FIR filter of fractional group delay and phase delay”, Elektronika Konstrukcje Technologie Zastosowania, No 4, pp. 17-20, 2014.

[13] A. Ashafi and F. J. Harris, "A novel square-root Nyquist filter design with prescribed ISI energy”, Signal Process., vol. 93, pp. 2626-2635, 2013.

[14] F. J. Harris, K. C. Dick, K. Moerder and S. Seshagiri, "An improved square-root Nyquist shaping filter," in Proc. Software Defined Radio, SDR'05 (conference), pp. 15-17, 2005. 